\title{
2019-Ncov - Controls and Routes of Transmission in Dental Practice
}

\author{
Malika Jagannath Sehgal' ${ }^{1}$, Surekha Dubey (Godbole) ${ }^{2}$
}

1, 2 Department of Prosthodontics, Sharad Pawar Dental College and Hospital, Sawangi, Maharashtra, India.

\section{ABSTRACT}

\section{BACKGROUND}

Since the emergence of $\mathrm{nCoV}-19$, till date it has affected several countries. This disease which is now called COVID-19 [(termed by World Health Organization (WHO)] is caused by a novel coronavirus, labelled as severe acute respiratoy syndrome-Coronavirus-2 (SARS-CoV-2) (termed by International Committee on Taxonomy of Viruses). Belonging to the family of Coronaviridae, of the order Nidovirales, it comprises of large, solitary, plus-stranded ribo nucleic acid (RNA) in their genome. The 2019-nCoV explored in Wuhan belongs to the $\beta-\mathrm{CoV}$ according to the phylogenetic analysis based on the viral genome, and it chiefly infects the respiratory, gastrointestinal, and central nervous system of humans and mammals. Its primary means of spread is either directly or indirectly by the means of droplets via the respiratory tract and fomites respectively. The most common routinely used instruments in the field of dentistry include airotors in the form of rotatory instruments and other surgical instruments. In the meantime, these instruments produce aerosols in the form of both larger and smaller droplets which generally consist of saliva, blood, microorganisms and other debris. Therefore due to possible aerosol and droplet production in the dental profession, $\mathrm{nCoV}-19$ poses a significant risk of infection transmission. To combat $\mathrm{nCoV}-19$, it is essential for a dental clinician to finely tune the protective approaches by concentrating towards the patient safety, personal protective gear and maintaining the hand hygiene. In addition to its modes of diffusion in dentistry, some other important themes like management of the patient, patient instructions and guidelines that a dentist should follow in day to day practice have been raised in this review.

\section{KEY WORDS}

2019-nCov; Transmission; Dental Guidelines; Epidemiology
Corresponding Author: Dr. Malika Jagannath Sehgal, Department of Prosthodontics, Sharad Pawar Dental College And Hospital, Sawangi, Maharashtra, India.

E-mail: malikasehgal123@gmail.com

DOI: $10.14260 / \mathrm{jemds} / 2021 / 642$

How to Cite This Article:

Sehgal MJ, Dubey S. 2019-Ncov - controls and routes of transmission in dental practice. J Evolution Med Dent Sci 2021;10(36):3159-3165, $10.14260 /$ jemds/2021/642

Submission 24-02-2021,

Peer Review 03-08-2021

Acceptance 10-08-2021,

Published 06-09-2021.

Copyright (C) 2021 Malika Jagannath Sehgal et al. This is an open access article distributed under Creative Commons Attribution License [Attribution 4.0 International (CC BY 4.0)] 


\section{EPIDEMIOLOGY}

At the end of the year in early December or even as early as late November 2019, Wuhan located in the Hubei province of China reported numerous patients suffering from pneumonia like symptoms whose etiology were yet to be discovered. These cases were more likely linked to the Huanan seafood wholesale market selling wild and live animals. ${ }^{1}$ With the drastic increase in the number of individuals experiencing similar symptoms, The public health experts of Wuhan decided to cease the activities in the market on January 1, $2020 .^{2}$ The National Establishments in China informed World Health Organisation about the current outbreak on January 3 2020 , stating that an aggregate of 44 patients suffered from such pneumonia. In the course of this reporting period, the agent causing this pneumonia was not recognized. ${ }^{3}$ Well ahead on January 7, 2020 a novel category of coronavirus was identified by the Chinese authorities. For diagnosing this novel coronavirus, specific diagnostic kits are required which are based on the genetic sequence of the former. Therefore on 12 January 2020, China revealed the sequence which thus enabled other countries to develop the diagnostic kits for the testing purpose.

On January 13 and 15, 2020, The Community Health Department of Thailand and The Ministry of Health, Labour and Welfare, Japan affirmed their first cases of a novel coronavirus, imported all the way from Wuhan respectively. ${ }^{3}$ At the end of the January that is on the $30^{\text {th }}$ COVID-19 was declared as an epidemic and a public health emergency of international concern. ${ }^{2}$ The current outbreak was recognized as a pandemic on 11 March 2020.4

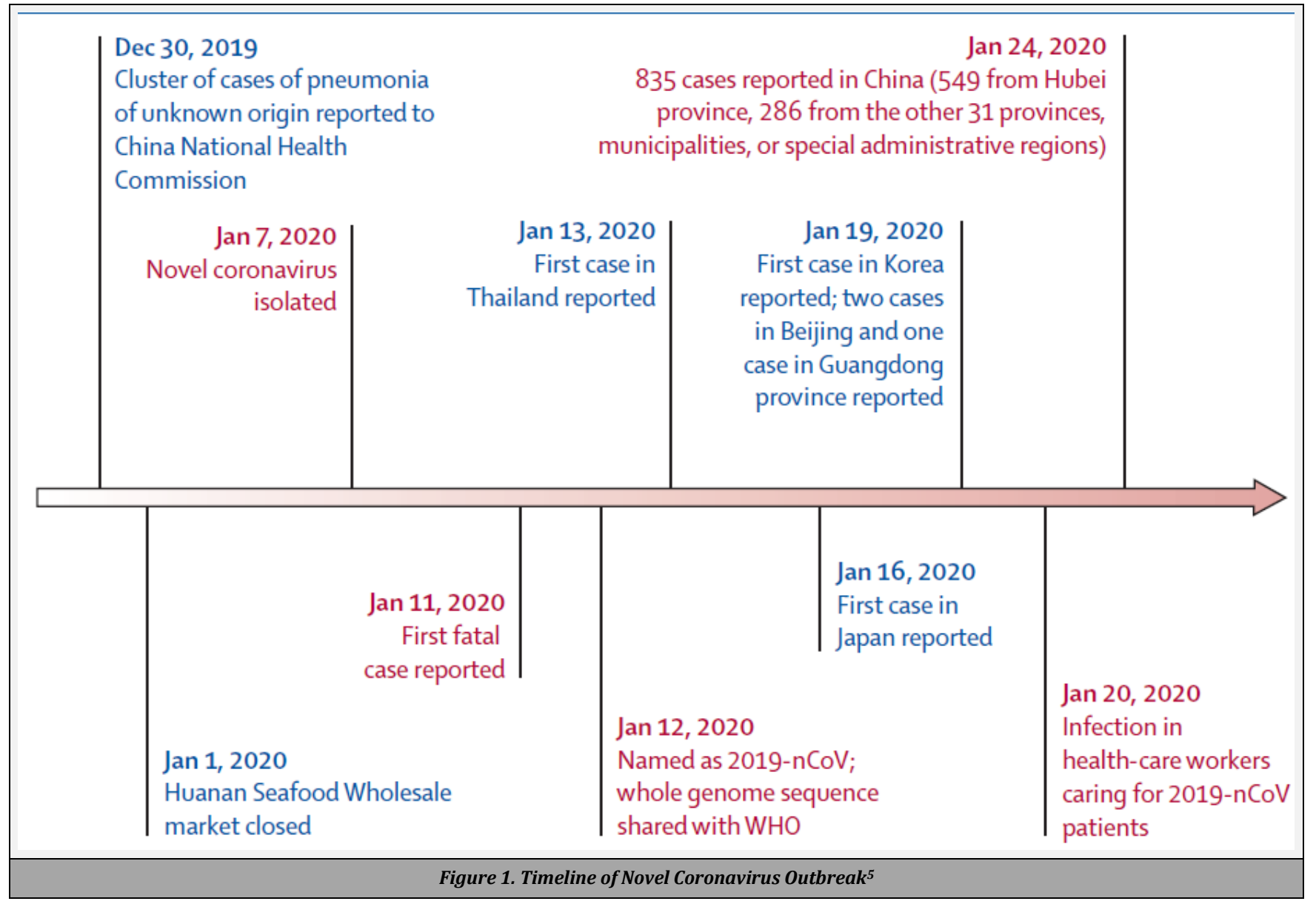

\section{CURRENT SITUATION}

Data as reported by World Health Organisation national authorities by 3:13 p.m. CEST, 22 September, 2020 estimates universally $31,174,627$ confirmed cases of novel coronavirus leading to 962,613 deaths with a global level of risk being very high affecting 213 countries, areas or territories. Till date in India from January 3 to 3:13 p.m. CEST, 22 September 2020, there have been a total of 5,562,663 confirmed cases that has led to 88,935 deaths. ${ }^{6}$ The occupational safety and health administration places dental health care worker in an exceedingly elevated peril section, due to the type of occupation which increases their odds of acquiring viral infection from a recognized or a suspected individual in the course of carrying out particular measures. ${ }^{7}$ According to The New York Times, dentists face the greatest novel coronavirus risk (figure-2). ${ }^{8}$ Thus, dental patients and specialists can possibly be subjected to multiple infective microbes, together with viruses and bacteria that pass on a disease to the oral cavity and respiratory tract. There is a consistent high risk of carrying 2019-nCoV infection in dental care settings which is mainly as a consequence of the particularities of its techniques involving uncompromising face-to-face confrontation with patients through everyday disclosure of fluids that are not only of oral in origin but also the other biological liquids, besides the usage of sharp instruments. ${ }^{9}$ 


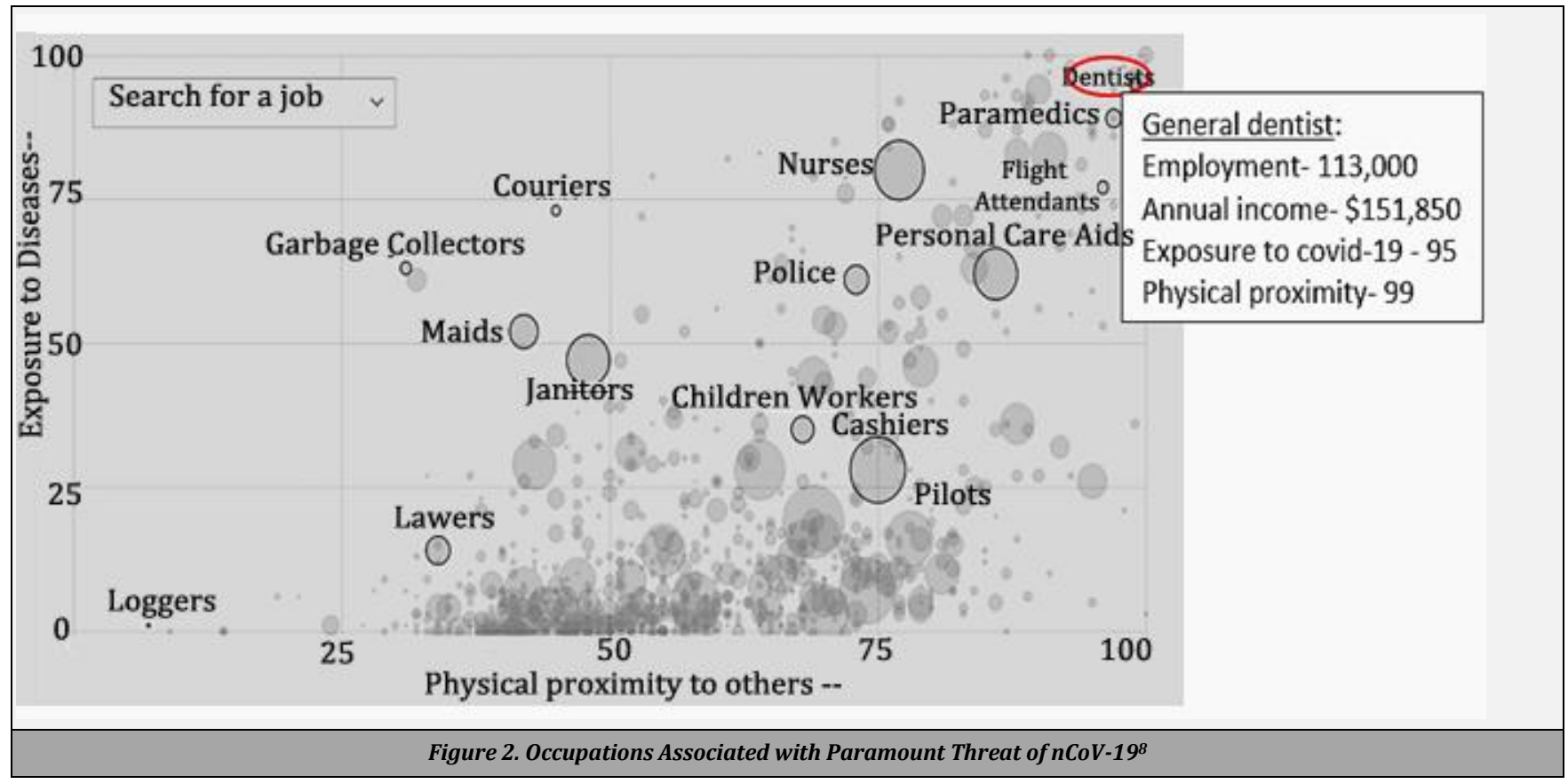

\section{AETIOLOGY}

This disease which is now called COVID-19 (termed by World Health Organisation) is caused by a novel coronavirus, labelled as SARS-CoV-2 (termed by International Committee on Taxonomy of Viruses). Belonging to the family of Coronaviridae, of the order Nidovirales, it comprises of large, solitary, plus-stranded RNA in their genome which was discovered through whole genome sequencing, polymerase chain reaction (PCR) and culture of bronchoalveolar lavage fluid derived from affected patients. The coronavirus subfamily is divided into alpha-, beta-, gamma- and delta-. However, the 2019-nCoV explored in Wuhan located in the Hubei province of China belongs to the $\beta-\mathrm{CoV}$ according to the phylogenetic analysis based on the viral genome, and it chiefly infects the respiratory, gastrointestinal, and central nervous system of humans as well as mammals. ${ }^{10}$

Being the seventh coronavirus, COVID-19 has $75-80 \%$ genomic resemblance to the severe acute respiratory syndrome coronavirus and $50 \%$ to the middle east respiratory syndrome coronavirus (MERS-CoV) and utilizes the same cell receptor, angiotensin-converting enzyme II (ACE2), that is used by SARS-CoV. ${ }^{11}$ A similarity in the genomic nucleotide sequence was acknowledged between a coronavirus (BatCoV RaTG13) detected in the bat rhinolophus affinis from Yunnan Province, China, and 2019-nCoV. Likeness of $96.2 \%$ was observed, thereby, indicating that the natural host of the novel coronavirus may also be the rhinolophus affinis bat. Nevertheless, the difference in the dissimilar genomic sequence may as well suggests the existence of one or more intermediate hosts between the bat and human ${ }^{12}$ as established by Liu and his colleagues from the Guangdong wildlife rescue center of China on 24 October 2019. They obtained SARS-CoV like CoV from the samples they attained from the lung of two dead Malayan pangolins. The lungs of these supposed intermediate host was filled with frothy liquid and exhibited pulmonary fibrosis. This information was discovered close to when the COVID-19 outbreak occurred, therefore concluding that Pangolin-CoV genome showed
91.02\% nucleotide identity with the SARS-CoV-2 genome and may be the midway host of 2019-nCoV.13

Source of Transmission and Incubation Period The principal manner of spread of novel coronavirus can occur by any of the two means that is either directly or indirectly. The direct spread ensues by the means of aerosols or droplets while the indirect spread take place by way of fomites. The average incubation period ranges for about 3 - 14 days. However, the reproduction number (Ro) that is the parameter which designates exactly how communicable an infective disease is, in general alleged to lie midst 2.0 and 2.8. According to one of the research, the high viral burden was detected in symptomatic patients almost immediately after the commencement of the symptoms, with highest viral load in nose followed by throat. The viral liability in symptom-free together with symptomatic patients were quite similar. ${ }^{14}$

\section{Signs and Symptoms}

nCoV-19 is a respiratory infection, that in the majority of the disease ridden individuals progresses from demonstrating mild to moderate manifestations. However, many patients pull through it without necessitating any extraordinary management. However, the ones exhibiting underlying comorbidities and individuals above 60 years of age will unveil amplified risk of evolving into debilitating ailment and even fatality as well as nosocomial infections. ${ }^{15,16}$

\section{General Manifestations}

- Fever- in 80 - $99 \%$ individuals

- Tiredness- $44-70 \%$

- Dry cough- 48 - $76 \%$

\section{Additional Manifestations}

- Breathing difficulty- $30-55 \%$

- Physical discomfort- $44-70 \%$

- Sore throat 
- A small number of individuals will document loose bowels, nausea or rhinorrhea. ${ }^{15,17}$

\section{Diagnosis}

For the purpose of diagnosis, the nasopharyngeal or oropharangeal swabs are taken from the superior part of the respiratory area. Nevertheless this method of gathering the samples involves end-to-end contiguity amongst the healthcare employee and the patient which pretences a substantial threat of viral diffusion from the patient to the one accumulating the samples.

Likewise, the major disadvantage of using this method of sample collection is that it causes patient uneasiness and may possibly elicits haemorrhage particularly in those individuals demonstrating blood dyscrasias. To overcome this shortcoming, a non-invasive method of sample collection is devised where in sputum originating from the inferior respiratory area is collected and is used for the purpose of diagnosis. However, only $28 \%$ of $\mathrm{nCoV}-2019$ cases produces sputum of investigative importance. The procedure of sample collection is relatively simple wherein the patient is requested to spew in a sterilized ampoule. Since, this procedure do not require a close contact between the healthcare employee and the patient, the chances of viral dissemination is comparatively low. 18

\section{Modes of Transmission of an Infection in a Clinical Setup}

Dental clinicians being placed in an elevated hazard category are not only at the risk of getting exposed to COVID-19 but also retain a threat to other viral infections like hepatitis $B$, human papilloma virus (HPV), human herpes virus, etc. ${ }^{19,20}$ Use of rotary and surgical instruments such as high velocity handpieces, ultrasonic tips and the two-way or three-way syringes have always been a fundamental part of the dental practice. Such tools are capable of generating an apparent splatter not only comprising of saliva, water droplets, blood, remnants produced during a dental treatment but also numerous pathogens. This perceptible spray settles promptly after journeying for a small range thus coming in contact with the ground, adjoining working surfaces, dental clinician and the patient. The modes of infection transportation are listed below-

\section{Airborne Spread}

Dental patients may indulge in the activity of coughing, sneezing, laughing, talking or receiving dental conduct particularly with regard to the use of a high velocity handpiece and ultrasonic devices. Performing such activities or undergoing such a conduct leads to the aerosolization of the secretions of oral cavity. ${ }^{21}$ Such an individual tends to produce both larger $(>5 \mu \mathrm{m}$ in diameter) and smaller $(\leq 5 \mu \mathrm{m}$ in diameter) droplets or aerosols. ${ }^{22}$ An aerosol is defined as an assemblage of concrete or fluid elements hanging in the midair. ${ }^{23}$ Due to the gravitational pull, the droplets which are larger in size are quick to fall on the ground, thereby concluding that larger droplet conduction necessitates neighbouring corporeal vicinity amongst the ill individual and a vulnerable contact. According to World Health Organisation, the droplets or particles which are smaller in diameter have the propensity to settle down slowly and may remain suspended in the environment for an extended period of time. Thus, allowing the infection to propagate lengthier distance. These particles can ascend either from nasopharynx or oropharynx and are frequently accompanied with saliva. While the droplets which are greater in diameter may perhaps participate in viral conduction in the immediate vicinity. ${ }^{10}$

It is physically conceivable for a pathogen to diffuse following an aerosol route only:

1. If the source of aerosol is derived from a contagious individual.

2. If the sustainability of the pathogen in the surroundings is for an extended period of interval.

3. If the purposed tissues is accessible to the infectious agents.

In order to combat nCoV-19, it is essential for a dental clinician to finely tune the protective approaches by concentrating towards the patient safety, personal protective gear and maintaining the hand hygiene. ${ }^{24}$

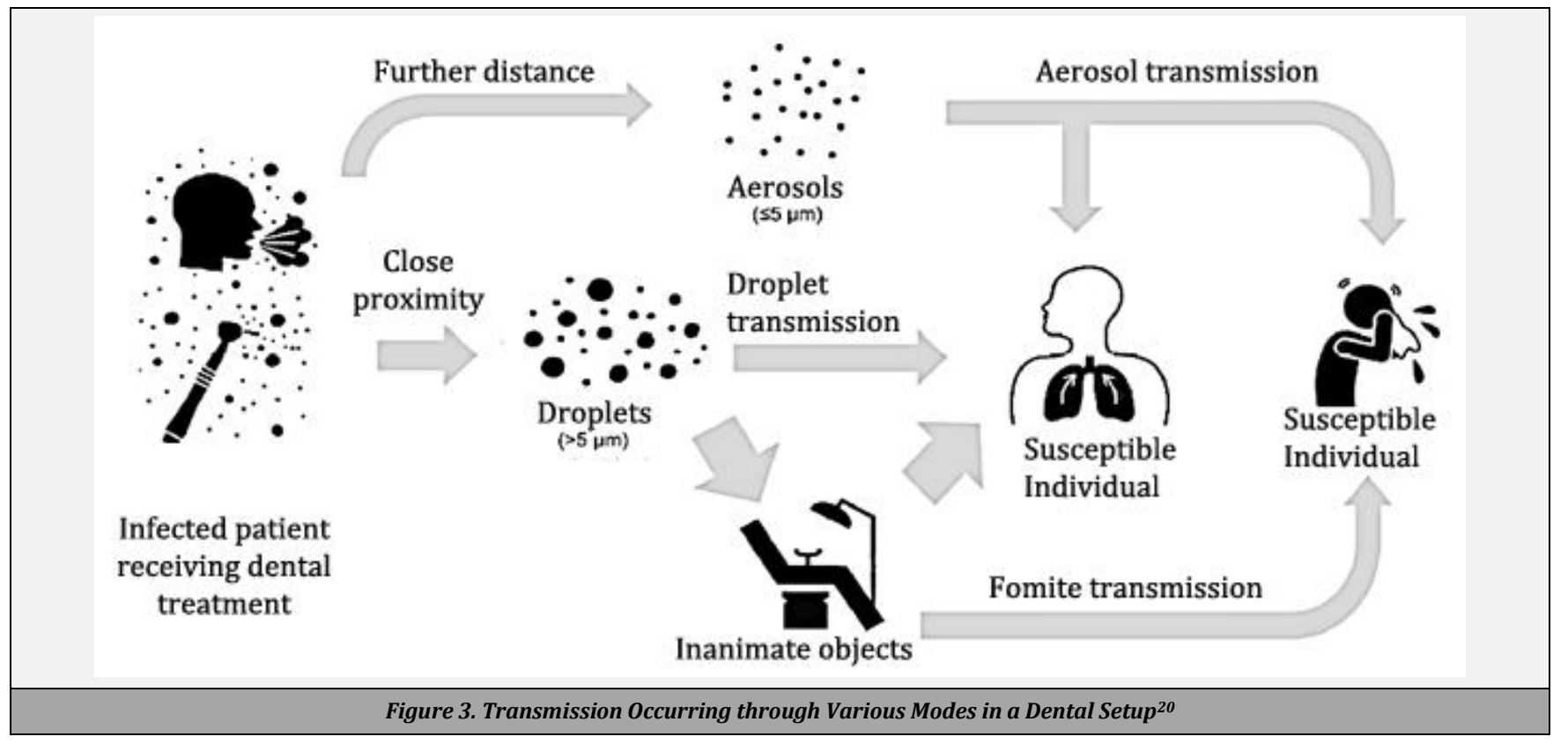


When a dental professional uses a high speed handpiece for performing dental procedures, a large amount of heat is produced as a result of friction between the tooth surface and the rotating bur. Hence, to minimize this, a coolant in the form of water is required to reduce the temperature upsurge since too much heat can bring about pathological changes in dental hard tissues including the dental pulp. This water coolant nevertheless produces aerosols, which when combined with oral fluids such as blood or saliva leads to the production of bioaerosols. $^{22}$

Therefore, it becomes challenging to escape the propagation of hefty aggregates of aerosol and droplet variegated with patient's saliva and even other oral fluids throughout the dental practice. Besides the infested sufferers of cough and breath, dental apparatus such as high velocity dental handpiece which utilizes the high-speed gas to drive the turbine also leads to extended production of bioaerosols. ${ }^{12}$

\section{Contact Spread}

A dentist may come in contact with the patient either directly or indirectly. Direct contact can be through oral fluids including blood while the indirect contact includes touching contaminated dental instruments or touching the surfaces like handle of the dental chair that the patient has touched, becomes a possible route for the transmission of virus. ${ }^{12}$ Few studies have shown that this virus has the tendency to survive in the aerosols and on the superficial surfaces for hours and days respectively. Recent evidences have also revealed that the transmission of the virus can even ensue through an asymptomatic patient. ${ }^{7}$ Allocation of the pathogenic agent to the mouth, nose or eyes can take place through contaminated hands contacting the former thus, giving rise to a fresh case of contagion..$^{22}$

\section{PATIENT MANAGEMENT}

Patient screening is one of the key factor in diagnosing not only symptomatic patients but also the asymptomatic patients. During a routine dental check-up, the dental professional should take a thorough medical history along with the list of medications that the patient is consuming. Targeted questionnaire should be formed asking the relevant info such as recent travel history, fever, symptoms of upper and lower respiratory tract or close contact with those who have travelled from the suspected regions as well. Not to be specific but the fever and fatigue can also occur due to acute dental conditions. Hence, the etiology should be confirmed first before arriving at any supposition. Treatment should be carried out for patients falling under the following categories-

\section{A. Those Requiring Emergency Treatment}

1. Patients with unstable maxillomandibular fractures that can compromise the patient's airway.

2. Intraoral or extraoral diffuse swellings that are of soft tissue in origin which can lead to airway obstruction.

3. Uninhibited post-operative bleeding.

B. Those That Can Be Managed without Aerosol Production

1. Patients suffering from severe tooth pain and inflammation requiring extraction of the culprit tooth.

2. Individuals exhibiting fracture of vital tooth.

3. Avulsed or luxated tooth.

4. Those suffering from dry socket.

5. Pericoronitis.

6. Localised dental abscess.

7. Those suffering from soft tissue laceration due to broken or defective orthodontic appliances

8. Recementation of the debonded prosthesis

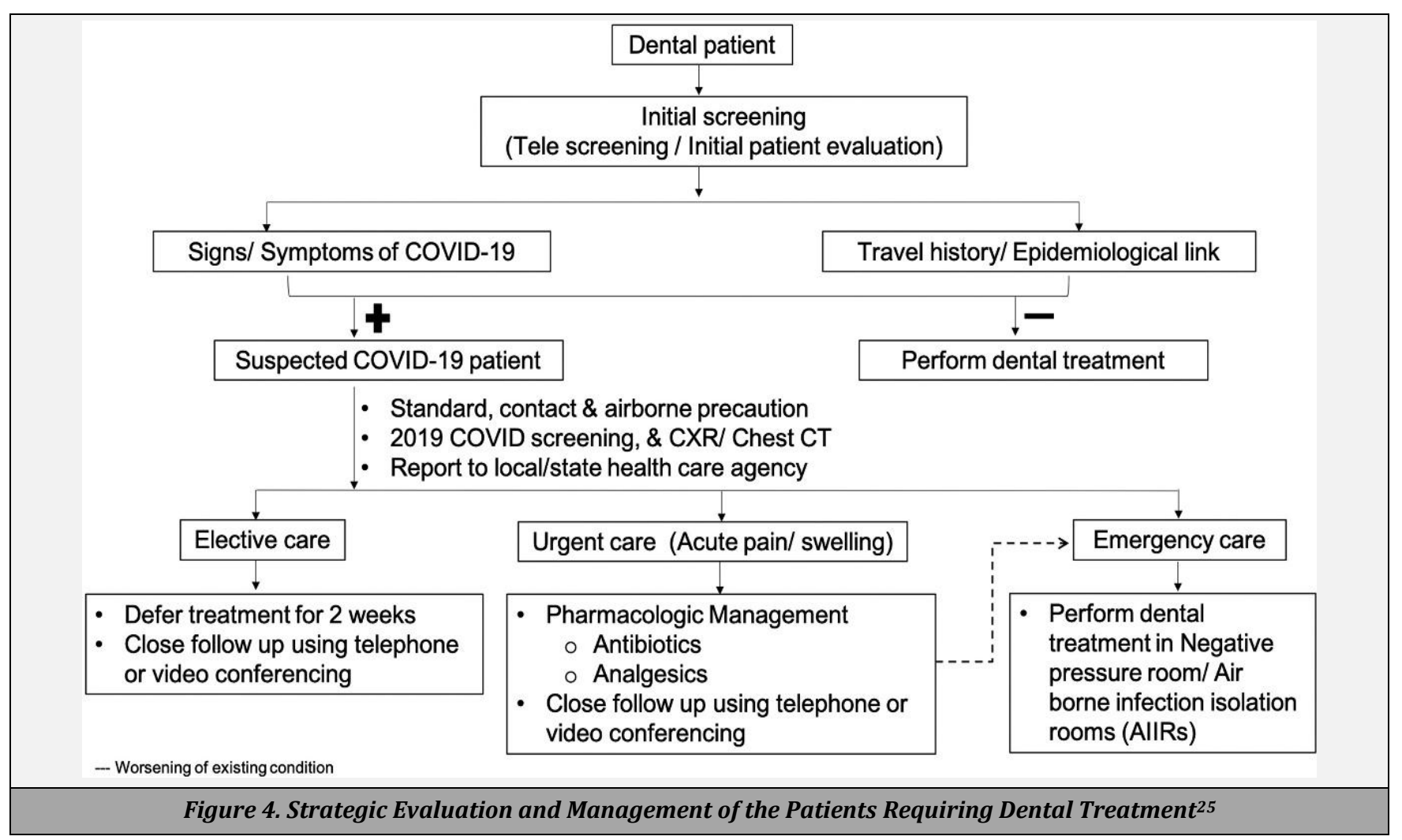


C. Those Requiring Urgent Treatment That Cannot

Be Managed without Aerosol Production

1. Individuals suffering from severe tooth pain and pulpal inflammation requiring aerosol production.

2. Patients exhibiting fracture of the vital tooth that cannot be managed without aerosol production

3. Cleaning of the fixed debonded prosthesis requiring temporary cementation. ${ }^{23}$

4. Removable dental prosthesis adjustments for the patients undergoing radiation therapy.

5. Acute pain of endodontic or periodontal in origin.

\section{Patient Instructions}

Wearing a mask should be mandatory for all the patients, staff present in the dental office as well as for the operator. Instruction pamphlets regarding the coughing and sneezing etiquette should be pasted in the waiting room. Former will ensure that all the patients coming to the dental clinic are covering their mouth and nose while performing these activities. A yellow bin should be kept in the waiting room to warrant that the patients are disposing off their infectious tissues in them, followed by hand hygiene. These bins should be adequately labelled so that it becomes easy for the patients to know its purpose. Adequate ventilation should be maintained. Patients should be made to sit with a distance of approximately 1 meter between them. ${ }^{22}$

According to CDC (Centre for Disease Control and Prevention) a dentist should follow guidelines:

1. Priority should be given to the emergency or the urgent procedure only. By executing these solitary measures, supplies such as personal protective equipment (PPE) for the staff members as well as those required for the care of the patient can be conserved. These actions will also aid in escalating the existing hospital volume.

2. The ailing dental personnels should be awarded with the sick leaves, which should be pliable and in favour of the health of the personnel. Those suffering from the symptoms indicating respiratory infection and the ones who develop the warning sign at work should be made to stay at home.

3. Screening of the patients experiencing respiratory symptoms such as cough, fever or difficulty breathing should be carried out telephonically. In case the patient turns up to be positive for the above mentioned symptoms, dental care should be averted. Whenever feasible, contingency dental management must be deferred until the patient pull over and is done with such an infection.

4. If dental management is postponed then the patient should be delivered an all-inclusive list of instructions and the suitable medications that can be followed at home. ${ }^{7}$

\section{Other Precautions}

- The procedures encompassing the use of such instruments which are capable of producing aerosols or droplets should be avoided. The dentists have been recommended to use saliva ejection device with high volume in order to shrink the aerosol generation. The animalcules in the aerosols can be curtailed by using a mouth rinse pre-operatively.

- High volume evacuator should be used. Aforementioned helps in removal of the aerosol and thus reduces the contamination. The only disadvantage of these evacuators is that it cannot be used by a clinician single handedly, thus four handed dentistry decorum needs to be pursued.

- While making of an impression in a COVID-19 patient, the right size of impression tray should be selected to prevent the cough reflex because of retching, which can arise as a result of inappropriate tray size.

- During the condition explicit procedures such as root canal therapy, complete denture try-in or during the delivery of any other prosthesis, a clinician should escape avoidable communication with other objects or surfaces in a dental clinic with the intention of lessening the transmission occurring through fomite route.

- Those patients reporting to the dental clinic with severe pain and are indicated for extraction, should be made to lie in a recumbent position. Above mentioned safety measure will enable the clinician to avoid working in the way of patients breathing space.

- Sophisticated standards of respiratory defence such as EUFFS respirators should be taken into consideration at the time of carrying out an emergency dental conduct on an alleged $n \mathrm{CoV}-19$ patient. ${ }^{22}$

- Application of a rubber dam arranges for a degree of barricade against the microorganisms present in the saliva or the aerosol generated during a dental conduct. According to Cochran et al. 1989 he concluded that the use of rubber dam reduces the spread of microorganisms by 90 $\%$.

Anti-retractive valves should be incorporated in the dental handpieces in order to circumvent the hazard of contamination and superinfection. ${ }^{26}$

\section{CONCLUSIONS}

With the emergence of COVID-19 outbreak, human race is facing not only major health risks but also the economic and mental challenges at a worrying rate. An improved understanding of the transmission characteristic of this infection and the intimidations that is being posed to the public health will help us better understand the nature of the disease. Aforementioned shall aid us not only in recognising the disease but also lay a hand in resolving the carelessness which may occur in day to day dental practices. Additionally in the era where prevention plays a crucial role, it should be the responsibility of every individual as well as healthcare professionals to follow all the necessary defensive measure such as wearing mask and inculcating proper hand hygiene to protect not just themselves but also those around them.

Financial or other competing interests: None.

Disclosure forms provided by the authors are available with the full text of this article at jemds.com.

\section{REFERENCES}

[1] Arabi YM, Murthy S, Webb S. COVID-19: a novel coronavirus and a novel challenge for critical care. Intensive Care Med 2020;46(5):833-6.

[2] Jiang F, Deng L, Zhang L, et al. Review of the clinical characteristics of coronavirus disease 2019 (COVID-19). J Gen Intern Med 2020;35(5):1545-9. 
[3] 20200121-sitrep-1-2019-ncov.pdf [cited 2020 Mar 27]. https://www.who.int/docs/defaultsource/coronaviruse/situation-reports/20200121sitrep-1-2019-ncov.pdf

[4] Bell DJ. COVID-19. Radiology Reference Article. Radiopaedia.org Radiopaedia. [cited 2020 Mar 28]. https://radiopaedia.org/articles/covid-19-3

[5] Wang C, Horby PW, Hayden FG, et al. A novel coronavirus outbreak of global health concern. The Lancet 2020;395(10223):470-3.

[6] Coronavirus [cited 2020 Apr 15]. https://www.who.int/emergencies/diseases/novelcoronavirus-2019

[7] CDC. Coronavirus Disease 2019 (COVID-19). Centers for Disease Control and Prevention. 2020 [cited $2020 \mathrm{Apr}$ 15]. https://www.cdc.gov/coronavirus/2019ncov/hcp/dental-settings.html

[8] Gamio L. The workers who face the greatest coronavirus risk. The New York Times. 2020 Mar 15 [cited 2020 Apr 6].

https://www.nytimes.com/interactive/2020/03/15/bu siness/economy/coronavirus-worker-risk.html

[9] Kampf G, Todt D, Pfaender S, et al. Persistence of coronaviruses on inanimate surfaces and their inactivation with biocidal agents. J Hosp Infect 2020;104(3):246-51.

[10] Fehr AR, Perlman S. Coronaviruses: an overview of their replication and pathogenesis. In: Maier HJ, Bickerton E, Britton P, eds. Coronaviruses: Methods and protocols. (Methods in Molecular Biology). New York, NY: Springer 2015: p. 1-23. https://doi.org/10.1007/978-1-49392438-7_1

[11] Arabi YM, Murthy S, Webb S. Correction to: COVID-19: a novel coronavirus and a novel challenge for critical care. Intensive Care Med 2020;46(5):1087-8.

[12] Peng X, Xu X, Li Y, et al. Transmission routes of 2019-nCoV and controls in dental practice. Int J Oral Sci 2020;12(1):9. http://www.nature.com/articles/s41368-020-0075-9

[13] Zhang T, Wu Q, Zhang Z. Probable pangolin origin of SARSCoV-2 associated with the COVID-19 outbreak. Curr Biol 2020;30(7):1346-51.e2.
[14] Zou L, Ruan F, Huang M, et al. SARS-CoV-2 viral load in upper respiratory specimens of infected patients. N Engl J Med 2020;382(12):1177-9.

[15] Coronavirus [cited 2020 Apr 5]. https://www.who.int/westernpacific/healthtopics/coronavirus

[16] Ganguly P, Yunus M, Khan A, et al. A study of nosocomial infection in relation to different host factors in an Indian teaching hospital. J R Soc Health 1995;115(4):244-6.

[17] Mackenzie JS, Smith DW. COVID-19: a novel zoonotic disease caused by a coronavirus from China: what we know and what we don't. Microbiol Aust 2020;MA20013.

[18] To KKW, Tsang OTY, Yip CCY, et al. Consistent detection of 2019 Novel Coronavirus in Saliva. Clin Infect Dis 2020;71(15):841-3. 18

[19] Khandelwal V, Khandelwal S, Gupta N, et al. Knowledge of hepatitis $B$ virus infection and its control practices among dental students in an Indian city. Int J Adolesc Med Health 2017;30(5).

[20] Gondivkar SM, Parikh RV, Gadbail AR, et al. Involvement of viral factors with head and neck cancers. Oral Oncology 2012;48(3):195-9.

[21] Meng L, Hua F, Bian Z. Coronavirus Disease 2019 (COVID19): emerging and future challenges for dental and oral medicine. J Dent Res 2020;99(5):481-7.

[22] Ge Z, Yang L, Xia J, et al. Possible aerosol transmission of COVID-19 and special precautions in dentistry. J Zhejiang Univ Sci B 2020;21(5):361-8.

[23] Jones RM, Brosseau LM. Aerosol transmission of infectious disease. J Occup Environ Med 2015;57(5):5018.

[24] Sabino-Silva R, Jardim ACG, Siqueira WL. Coronavirus COVID-19 impacts to dentistry and potential salivary diagnosis. Clin Oral Investig 2020;24(4):1619-21.

[25] Alharbi A, Alharbi S, Alqaidi S. Guidelines for dental care provision during the COVID-19 pandemic. Saudi Dent J 2020;32(4):181-6.

[26] Spagnuolo G, De Vito D, Rengo S, et al. COVID-19 outbreak: an overview on dentistry. Int J Environ Res Public Health 2020;17(6):2094. 